\title{
Hard X-ray Patterns of CME-Related Flares
}

\author{
Y. P. Li and W. Q. Gan \\ Purple Mountain Observatory, Chinese Academy of Sciences, Nanjing 210008, China \\ email: yplee@pmo.ac.cn
}

\begin{abstract}
The RHESSI hard X-ray spectra are investigated for a total of 23 CME-related flares. It is found that about $17 \%, 70 \%$, and $13 \%$ of the samples can be attributed to type A, $\mathrm{B}$, and $\mathrm{C}$, respectively. These ratios are not significantly different from those obtained using the data of HXRBS/SMM, although the ratio of type $\mathrm{C}$ in our CME-related flares is a little higher. More samples are obviously necessary to study the difference of hard X-ray spectra between the CME-related flares and non-CME-related flares.
\end{abstract}

Keywords. Sun: CMEs, flares, X-rays

\section{Introduction}

The relationship between solar flares and coronal mass ejections (CMEs) is an important topic and has been extensively studied. At present it seems to be clear that part of flares has an associated CME. We call this kind of flares as CME-related flares. Harrison (1995) indicated that higher intensity events are more likely to be accompanied by a CME, but this is not always the case (Green et al. 2002). Recently, Andrews (2003) made a statistical study based on a total of 229 M-class and X-class flares occurred from 1996 to 1999, which had a corresponding LASCO observations. He found that all the 14 $\mathrm{X}$-class flares in his samples have an associated CME, while there are $42 \%$ of M-class flares which have not any associated CME. The CME-related flares and non-CME-related flares seem to be independent of the positions where they happened on the solar disk.

The soft X-ray characteristics of CME-related flares and non-CME-related flares have been investigated by Kay et al. (2003). A comparison of flare parameters for a total of 69 flares indicates that there are systematic differences in the relationship between the peak intensity and duration, peak intensity and temperature for the two types of event. It was shown that higher intensity events are of longer duration in the case of CMErelated flares, and that the CME-related flares tend to have lower peak temperatures than non-CME-related flares of the same intensity.

However, so far there does not seem to have any detailed work on the hard X-ray characteristics of CME-related flares. Different hard X-ray patterns would implicate different environment where the energetic electrons are accelerated. Do the CME-related flares tend to be in conjunction with the topology of the open magnetic field? Do the nonCME-related flares tend to be in conjunction with the topology of the closed magnetic field? Statistical study of hard X-ray spectra for the CME-related flares would be helpful to address this kind of problems.

The hard X-ray patterns of solar flares were divided into three types (e.g., Tanaka 1987): type A or hot thermal flares, type B or impulsive flares, and type $\mathrm{C}$ or gradualhard flares. The detailed descriptions about this classification can also be found in Dennis (1988). Kosugi et al. (1988) made a statistical study for a total of 416 events observed with HXRBS/SMM. They found that among 416 events, 65 belonged to type A, 338 belonged to type B, and 13 belonged to type C. Cliver et al. (1986) noted that most type C flares are accompanied by CMEs. 
Table 1. CME-related flares and their hard X-ray types

\begin{tabular}{ccccc}
\hline Types & Date of flares/time & & \\
\hline A & Feb-21-02/12:26 & Feb-06-03/03:49 & Apr-24-03/12:53 & Apr-27-03/15:32 \\
B & Feb-20-02/09:59 & Apr-21-02/01:51 & Aug-03-02/19:07 & Aug-22-02/01:57 \\
& Nov-09-02/13:23 & Nov-10-02/03:21 & Apr-23-03/01:06 & Apr-26-03/08:07 \\
& May-27-03/23:07 & May-29-03/01:05 & Jun-09-03/11:28 & Jun-17-03/22:55 \\
& Oct-19-03/16:50 & Nov-01-03/22:38 & Nov-02-03/17:25 & Nov-03-03/09:55 \\
C & Jul-23-02/00:35 & Oct-24-03/02:54 & Oct- 28-03/11:10 & \\
\hline
\end{tabular}

The RHESSI (Lin et al. 2002) provides us an unprecedented chance to study the hard $\mathrm{X}$-ray spectra with a high energy resolution. This paper is to study the hard X-ray spectra of CME-related flares using the RHESSI data.

\section{Data analysis}

The CME lists up to December 2003 were taken from the website of http://lascowww.nrl.navy.mil/cmelist.html. Among the lists a total of $86 \mathrm{CMEs}$ which have a corresponding flare were preliminarily selected. Then we checked the availability of RHESSI observations from these preliminary candidates. Excluding the events with incomplete time coverage and the relatively weak emissions up to $100 \mathrm{keV}$, at last we selected a total of 23 events with which we can properly analyze the hard X-ray spectra.

We used the standard code SPEX to fit the hard X-ray spectra. The fitted model constitutes a thermal component plus two power-laws. In order to avoid the influence of some factors like lower energy cutoff (e.g., Gan et al. 2001) on the spectrum, only the power-law index of the higher energy end is taken into account. Table 1 presents the selected samples of CME-related flares and the derived types of hard X-ray patterns. As a matter of fact, the so-called soft-hard-harder spectral variations of type $\mathrm{C}$ did not appear in our samples. The type $\mathrm{C}$ flare in Table 1 is characterized by its long duration (>10 min.) and harder spectral index $(<3)$, the criterion as used by Kosugi et al. (1988).

From Table 1 we see that among a total of 23 CME-related flares, type A, B, and C are 4,16 , and 3 , taking the ratio of about $17 \%, 70 \%$, and $13 \%$, respectively. In comparison with the results by Kosugi et al. (1988), the ratio of type C pattern in CME-related flares seems to be a little higher than that obtained in spite of CME-related or not, where the ratio is only $3 \%$. However, both the small number of samples and the complex relationship between flares and CMEs prevent us to make a definite conclusion. Further studies should be based on more samples.

\section{References}

Andrews, M. D. 2003, Solar Phys. 218, 261.

Cliver, E. W. et al. 1986, ApJ 305, 920

Dennis, B. R. 1988, Solar Phys. 118, 49

Gan, W. Q., Li, Y. P., \& Chang, J. 2002, ApJ 552, 858

Green, L. M. et al. 2002, Solar Phys. 205, 325

Harrison, R. A. 1995, Astron. Astrophys. 304, 583

Kay, H. R. M. et al. 2003, Astron. Astrophys. 400, 779

Kosugi, T. \& Dennis, B. R. 1988, ApJ 324, 1118

Lin, R. P. et al. 2002, Solar Phys. 210, 3

Tanaka, K. 1987, PASJ 39, 1 